\title{
Parâmetros Doppler velocimétricos das artérias renais e da aorta abdominal em gatos da raça persa
}

\author{
Doppler velocimetric parameters of aorta and renal arteries of Persian cats
}

\author{
Cibele Figueira Carvalho $^{I^{*}}$ Giovanni Guido Cerri' ${ }^{\mathrm{II}}$ Maria Cristina Chammas ${ }^{\mathrm{II}}$
}

\section{RESUMO}

Foi realizado um estudo prospectivo com 50 unidades renais de gatos adultos jovens da raça persa, as quais foram examinadas ao ultrassom Doppler. Os animais foram selecionados como sadios por meio de exames laboratoriais e de pressão arterial sistólica. Foram mensurados os seguintes parâmetros: a velocidade de pico sistólico máximo (VPS), a velocidade diastólica mínima (VDF) e os índices de resistividade (IR) das artérias renais (AR) e da aorta (AO). Para a AR esquerda, foram obtidos VPS 40,96 $\pm 9,08 \mathrm{~cm} \mathrm{~s}^{-1}$ e IR $0,55 \pm 0,07$ e, para a AR direita, VPS $41,39 \pm 9,89 \mathrm{~cm} \mathrm{~s}^{-1}$ e IR $0,52 \pm 0,07$. Não foi observada diferença estatisticamente significante entre os lados. Assim, ao serem consideradas ambas as AR, obteve-se a média de VPS $41,17 \pm 9,40 \mathrm{~cm} \mathrm{~s}^{-1}$ e IR $0,53 \pm 0,07$. A média do diâmetro da $A R$ foi de $0,15 \pm 0,02 \mathrm{~cm}$. Para a aorta, obteve-se VPS $53,17 \pm 13,46 \mathrm{~cm} \mathrm{~s}^{-1}$ e média de diâmetro de 0,38 $\pm 0,04 \mathrm{~cm}$. Correlacionando-se os valores de VPS da AR e da AO, obteve-se um índice mínimo de 0,38 e

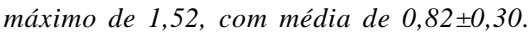

Palavras-chave: Doppler, rim, gatos.

\section{ABSTRACT}

Prospective study was made performing Doppler ultrasonographic exams of fifty kidneys belonging to Persian adult cats. The animals were classified as healthy by laboratorial exams and measurement of arterial systolic pressure. Maximal systolic blood flow velocity (VPS), minimal diastolic blood flow velocity (VDF) and resistivity index (IR) of renal arteries (AR) and aorta (AO) of each animal were evaluated. The results are: left renal artery VPS $40.96 \pm 9.08 \mathrm{~cm}$ $\mathrm{s}^{-1}$ and IR 0.55 \pm 0.07 ; right artery VPS $41.39 \pm 9.89 \mathrm{~cm} \mathrm{~s}^{-1}$ and IR $0.52 \pm 0.07$. It was not observed any statistical significant difference among the sides. Considering both renal arteries it was calculated VPS $41.17 \pm 9.40 \mathrm{~cm} \mathrm{~s}^{-1}$ and IR $0.53 \pm 0.07$. Renal arterial diameter was $0.15 \pm 0.02 \mathrm{~cm}$. The aorta VPS obtained was $53.17 \pm 13.46 \mathrm{~cm} \mathrm{~s}^{-1}$ and the diameter was $0.38 \pm 0.04 \mathrm{~cm}$. Correlation velocities of renal artery and aorta resuled in a relation 0.38 as minimal and 1.52 as maximal, with $0.82 \pm 0.30$.

Key words: Doppler, kidney, cats.

\section{INTRODUÇÃO}

As doenças renais têm alta morbidade em animais idosos, principalmente nos gatos. A ocorrência dessas afecções parece estar aumentando com a expectativa de vida dos animais de estimação. Além da idade avançada, fatores como alterações congênitas, alimentação inadequada, utilização de drogas nefrotóxicas e doenças infecciosas predispõem esses animais a lesões renais (NEWL et al., 1999). Na espécie felina, ainda é importante considerar-se a prevalência de doenças como a cardiomiopatia hipertrófica, em algumas raças, o hipertireoidismo e a hipertensão arterial sistêmica, pois são condições que interferem severamente na hemodinâmica renal e vice-versa. A deteç̧ão precoce dessas lesões depende do estudo da hemodinâmica renal. O dúplex Doppler colorido é uma técnica complementar à ultrassonografia convencional e à radiografia e tem numerosas

Instituto de Radiologia (INRAD), Hospital das Clínicas da Faculdade de Medicina da Universidade de São Paulo (HCFMUSP), 01402-030, São Paulo, SP, Brasil. E-mail: cibelefcarvalho@terra.com.br. Autor para correspondência.

IIFaculdade de Medicina, USP, São Paulo, SP, Brasil.

IIIServiço de Ultrassonografia, INRAD, HCFMUSP, São Paulo, SP, Brasil. 
aplicações na avaliação do aparelho urinário (CERRI et al., 1998; NEWL et al., 1999; NYLAND et al., 1993; PLATT, 1997; POLLARD,R. et al, 1999; RIVERS et al., 1997), pois é capaz de fornecer dados importantes para avaliar doenças renais devido à capacidade de estudarem-se detalhes da anatomia e da fisiologia renal por meio da análise dos fluxos sanguíneos, complementando as informações obtidas ao exame de ultrassonografia convencional (RIVERS et al., 1997). O exame ultrassonográfico com Doppler colorido e de amplitude é utilizado com freqüência na medicina humana com a finalidade de avaliar a arquitetura vascular renal (CERRI et al., 1998; PLATT, 1997). O mapeamento colorido permite observar a perfusão renal, selecionando-se rapidamente um vaso para análise espectral e coleta de dados sobre o fluxo sanguíneo. Embora a ultrassonografia seja um meio diagnóstico de rotina nos animais com doenças renais, é escassa a literatura internacional no que se refere à avaliação hemodinâmica dos vasos renais e abdominais que o ultrassom Doppler pode fornecer nesses casos. Valores de referência das velocidades de pico sistólico (VPS) e diastólica final (VDF) das artérias renais nos gatos ainda não foram descritos na literatura. Para a correta interpretação da capacidade diagnóstica do Doppler, é de fundamental importância que se conheça o perfil normal, bem como as eventuais mudanças fisiológicas.

Este trabalho teve como objetivo determinar parâmetros dopplerfluxométricos normais das artérias renais e da aorta abdominal em gatos adultos sadios da raça persa.

\section{MATERIAL E MÉTODOS}

O projeto foi submetido e aprovado pela Comissão de Ética do Instituto de Radiologia do Hospital das Clínicas da Faculdade de Medicina da Universidade de São Paulo. Foi realizado um estudo prospectivo de janeiro a dezembro de 2007. Nesse período, foram examinadas ultrassonograficamente 50 unidades renais, provenientes de 25 gatos da raça persa, 13 fêmeas e 12 machos, que apresentaram pesos corpóreos variados (com média de 4,8kg) e idade entre 12 e 60 meses (com média de 26 meses de idade). Todos os animais provieram de gatil de propriedade particular. Os animais foram selecionados como sadios por meio de exame clínico e exames laboratoriais (hemograma completo, bioquímica sérica para avaliação de função renal e hepática e urinálise tipo I) com valores considerados normais de acordo com o descrito na literatura (JAIN, 1993; KANEKO et al., 1997). Além disso, foi realizada medida de pressão arterial sistólica conforme preconizado em literatura (BROWN et al., 2007).
Foi utilizado um equipamento ultrassom Doppler da marca $\mathrm{GE}^{\circledR}$, modelo Logiq3, com transdutor multifrequencial linear de 7 a 10MHz. As imagens foram registradas em CD diretamente na mídia do aparelho. Todos os exames foram realizados por um único examinador no Instituto Brasileiro de Diagnóstico e Especialidades Veterinárias Provet, em São Paulo.

Os animais foram submetidos a jejum de sólidos no mínimo de oito horas e à ampla tricotomia abdominal para a realização dos exames ultrassonográficos. Para a avaliação ultrassonográfica, foi necessária a utilização de gel para contato em toda a região a ser examinada. $\mathrm{O}$ animal foi posicionado em decúbito dorsal, com o transdutor posicionado na parede lateral esquerda e direita do abdome, próximo à margem caudal das costelas. O exame foi realizado na seguinte seqüência: ultrassonografia modo-B, Doppler colorido e Doppler pulsado. Os rins inicialmente foram examinados ao ultrassom convencional, depois foram avaliados por meio de planos de cortes longitudinais, transversais e coronais e, por fim, foram avaliados quanto ao tamanho, à ecogenicidade e à relação córtico-medular, conforme estabelecido em literatura (BARR, 1990; BARR et al., 1990).

Após a ultrassonografia modo- $\mathrm{B}$, os rins foram avaliados por meio do mapeamento colorido para estudo da arquitetura vascular. Observaram-se as artérias renais após sua origem, próximo à aorta abdominal. Em seguida ao mapeamento colorido, o Doppler pulsado foi acionado, e o cursor foi posicionado na aorta, na região caudal à origem das artérias renais. Com a técnica bidimensional, identificouse cada uma das artérias renais, obteve-se um plano longitudinal e foi mensurado o diâmetro de cada vaso em questão. Usando o modo colorido foi possível determinar a presença ou ausência de fluxo no vaso (Figura 1). Por fim, o volume de amostra medindo entre 2 e $3 \mathrm{~mm}$ foi colocado na porção central do vaso e, por meio da técnica Doppler pulsado, obteve-se um traçado nessa região do vaso com, no mínimo, três ondas subsequentes. Após a correção do ângulo e com o traçado livre de artefatos, a imagem foi congelada, e foi realizada a análise da morfologia das ondas (Figura 2). Foram realizadas as medidas de VPS e VDF, e ainda foram calculados os IR desses vasos. A análise estatística dos resultados foi realizada com a aplicação de testes paramétricos, levando-se em consideração a natureza das variáveis e a variabilidade das medidas efetuadas. Cada uma das artérias recebeu tratamento estatístico em separado. Foram calculadas medidas de posição (mínimo, máximo, média e mediana) de cada um dos valores e dos índices estabelecidos. Foi necessário construírem-se intervalos de confiança para 


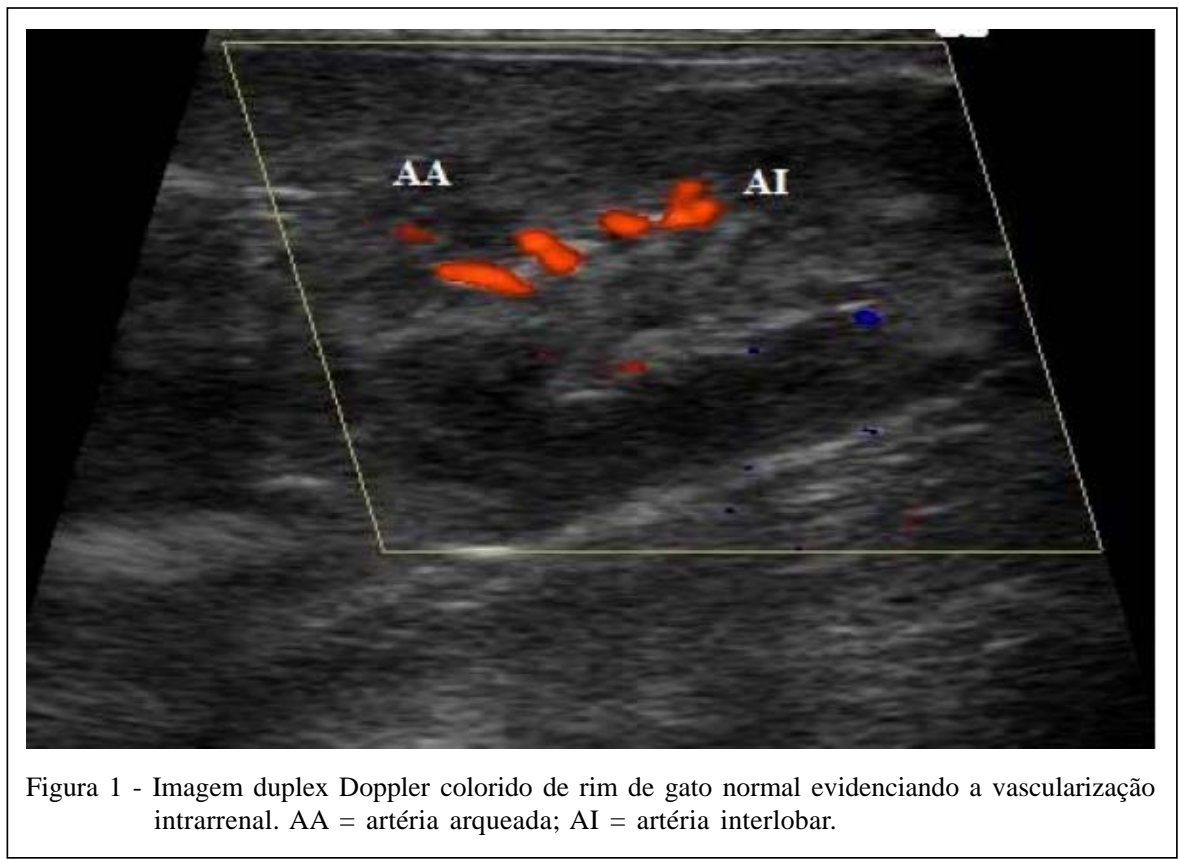

determinar o intervalo de valores plausíveis para os parâmetros de velocidade e índice de resistividade dos vasos avaliados, uma vez que não há limites estabelecidos em literatura. Foram calculados intervalos com nível de significância de 5\%. Foi utilizado teste $\mathrm{t}$ pareado para verificar se havia diferença significante entre as médias dos índices de resistividades e velocidades obtidos entre os lados. Verificou-se a presença de correlação entre dados utilizando o coeficiente de Pearson. Além disso, foram estabelecidos índices de relação entre as velocidades de pico sistólico das artérias renais e aorta.

\section{RESULTADOS}

O exame das 50 unidades renais revelou que a média do diâmetro da artéria renal foi de $0,15 \pm 0,02 \mathrm{~cm}$. Os dados obtidos foram dispostos em tabelas (Tabela 1). As tabelas 2 e 3 apresentam os valores médios das velocidades de pico sistólico (VPS), da velocidade diastólica final (VDF) e do fluxo sanguíneo e o índice de resistividade (IR) calculado das artérias renais direita, esquerda e de ambas. Para a artéria renal (AR) esquerda, foram obtidos os seguintes resultados: VPS $40,96 \pm 9,08 \mathrm{~cm} \mathrm{~s}^{-1}$ e IR $0,55 \pm 0,07$ e, para a artéria renal

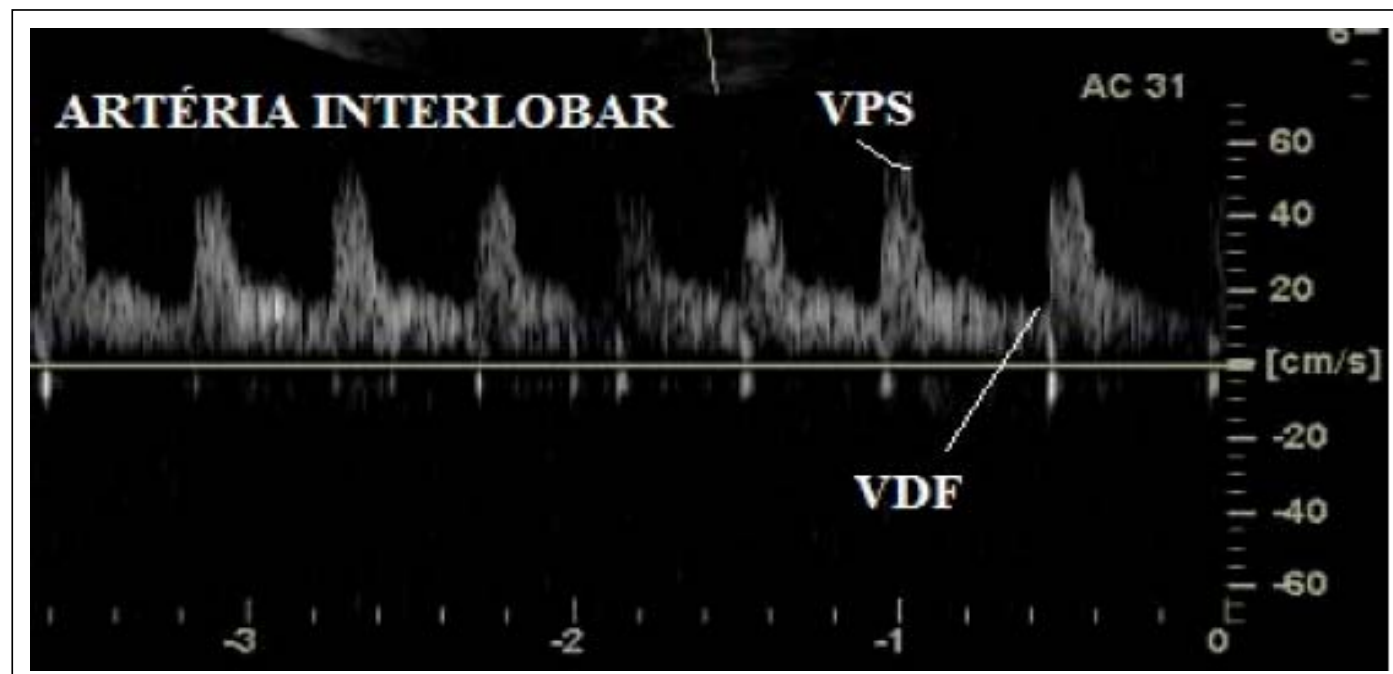

Figura 2 - Traçado espectral de artéria interlobar renal normal, apresentando morfologia de onda característica de fluxo arterial de baixa resistividade. VPS = velocidade de pico sistólico; VDF = velocidade diastólica final. 
Tabela 1 - Valores velocimétricos e diâmetros obtidos por meio do exame de ultrassonografia duplex Doppler das artérias renais de gatos adultos sadios da raça persa. São Paulo, SP, Brasil - 2007. (vps = velocidade de pico sistólico; vdf = velocidade diastólica final; ir = índice de resistividade).

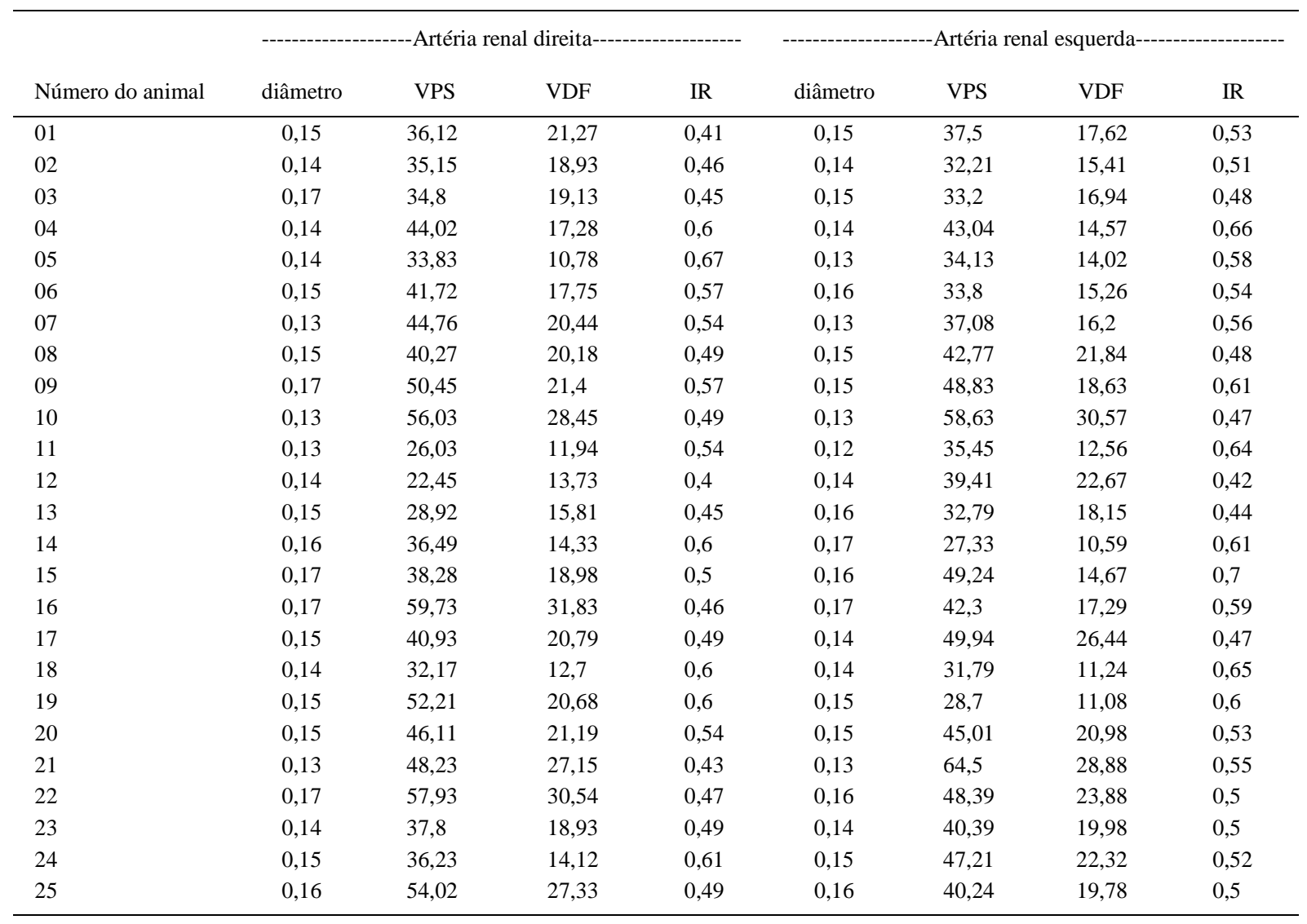

(AR) direita, foram obtidos VPS 41,39 $\pm 9,89 \mathrm{~cm} \mathrm{~s}^{-1} \mathrm{e} \mathrm{IR}$ $0,52 \pm 0,07$. Aplicando-se teste $t$ pareado, as médias dos valores de VPS e IR apresentaram similaridade entre os lados e não foi observada diferença estatisticamente significante. Ao serem consideradas ambas as artérias renais, foi obtida a média de VPS $41,17 \pm 9,40 \mathrm{~cm} \mathrm{~s}^{-1}$ e a média de IR de 0,53 $\pm 0,07$.

Tabela 2 - Médias e desvio padrão (dp) obtidos das velocidades de pico sistólico (vps) e da velocidade diastólica final (vdf) e índices de resistividade nas artérias renais (ar) nos lados direito e esquerdo. São Paulo, SP, Brasil 2007. n= número de amostra

\begin{tabular}{llll}
\hline & AR_VPS & AR_VDF & AR_IR \\
\hline Esquerdo & & & \\
Média & 40,96 & 18,46 & 0,551 \\
DP & 9,08 & 5,34 & 0,072 \\
N & 25 & 25 & 25 \\
Direito & & & \\
Média & 41,39 & 19,83 & 0,521 \\
DP & 9,89 & 5,69 & 0,073 \\
N & 25 & 25 & 25 \\
\hline
\end{tabular}

A tabela 4 apresenta os valores médios da VPS e VDF do fluxo sanguíneo e do diâmetro da artéria aorta abdominal em sua porção caudal à origem das artérias renais. Para aorta, foram obtidos VPS $53,17 \pm 13,46 \mathrm{~cm} \mathrm{~s}^{-1}$ e média de diâmetro de $0,38 \pm 0,04 \mathrm{~cm}$.

Com a utilização do coeficiente de Pearson, foi verificada a existência de correlações entre as VPS das artérias renais (direita e esquerda) e a VPS da aorta. Foi realizada a correlação entre essas velocidades e, nas relações artéria renal direita com aorta e artéria renal esquerda com aorta, não houve diferença estatisticamente significante. A média obtida da relação das VPS da artéria renal e aorta foi $0,828 \pm 0,296$, com valor mínimo de 0,387 e valor máximo de 1,518.

\section{DISCUSSÃO}

A literatura cita que nos gatos não há diferença estatisticamente significante entre os valores dos índices de resistividade encontrados em animais sedados e não-sedados (RIVERS et al., 1996; RIVERS et al., 1997). A literatura sugere que se adote o limite 
Tabela 3 - Média e desvio padrão (dp) obtidos dos diâmetros e das velocidades de pico sistólico (vps) e da velocidade diastólica final (vdf) das artérias renais (ar). São Paulo, SP, Brasil - 2007. n = número de amostra.

\begin{tabular}{lcccc}
\hline $\mathrm{N}=50$ & AR diâmetro $(\mathrm{cm})$ & AR VPS $\left(\mathrm{cm} \mathrm{s}^{-1}\right)$ & AR VDF $\left(\mathrm{cm} \mathrm{s}^{-1}\right)$ & AR IR \\
\hline Média & 0,15 & 41,17 & 19,14 & 0,54 \\
DP & 0,01 & 9,40 & 50 & 0,07 \\
Mínimo & 0,12 & 22,45 & 10,59 & 0,39 \\
Mediana & 0,15 & 40,26 & 18,93 & 0,53 \\
Máximo & 0,17 & 64,50 & 31,83 & 0,70 \\
\hline
\end{tabular}

superior de índice de resistência da artéria renal de 0,69 para os gatos (RIVERS et al., 1996). Outros estudos demonstraram valores diferentes destes, variando entre 0,70 e 0,71 . Neste estudo, os exames foram realizados com animais conscientes e ambientados com a sala. Além disso, todos tinham temperamento bastante cooperativo. Os índices de resistividade obtidos foram bem menores que os citados como referência pela literatura, com valores entre $0,54 \pm 0,07$. Considerandose que todos os animais foram selecionados como sadios e normopressóricos, pode-se sugerir que, devido ao temperamento dessa raça em especial, todos estivessem calmos. Assim, os valores obtidos são expressões da realidade. Os trabalhos apresentados na literatura não separam os pacientes por faixa etária. É conhecido que, na espécie humana, há uma tendência de aumento dos valores do IR em crianças e idosos (RIVERS et al., 1996; PLATT, 1997). Talvez isso possa acontecer também com os gatos; porém, há a necessidade de realizarem-se estudos comparativos com animais sadios em diversas faixas etárias.

A literatura cita que há uma tendência de redução dos valores de velocidades sistólica e diastólica e dos valores de IR dos ramos vasculares maiores para os menores (MELO et al., 2006). Concordando com essa proposição neste estudo, foram obtidos valores médios de velocidade sistólica na aorta maiores que nas artérias renais. Nos animais, é muito comum encontrar-se uma bifurcação ou mais da artéria renal antes da sua entrada na pelve (DYCE et al., 1997). Isso faz com que o volume sanguíneo de entrada seja dividido, diminuindo ainda mais o impacto de grandes velocidades de fluxo vindas do vaso-fonte. Não há referências na literatura quanto aos parâmetros normais da velocidade do fluxo sanguíneo nesses vasos nos gatos.

Os valores de VPS das artérias renais direita e esquerda não apresentaram diferença estatisticamente significante. Correlacionando-se os valores de VPS das artérias renais e da aorta, obteve-se um índice entre 0,38 e 1,52 ou $0,82 \pm 0,30$. Com este estudo, sugere-se a utilização dessa relação entre as velocidades sistólicas desses vasos a fim de auxiliar na monitoração dos parâmetros hemodinâmicos de animais normais. Não se conhece até o momento a existência de referências publicadas na literatura sobre os parâmetros de velocidade normal das artérias renais em gatos adultos sadios da raça persa. Além de ter-se estabelecido, neste trabalho, esses valores de referência, sugeriu-se a utilização de um índice de relação entre as velocidades sistólicas das artérias renais e da aorta. Além disso, a obtenção de valores de índices de resistividade mais baixos do que os citados na literatura até o momento sugerem a necessidade de mais estudos em animais com diferentes faixas etárias e em condições clínicas diferentes. Finalmente, é de fundamental importância a determinação de parâmetros normais que realizem com segurança e precocidade o diagnóstico das alterações hemodinâmicas que eventualmente possam preceder as alterações funcionais.

\section{CONCLUSÕES}

Determinou-se que os valores normais de velocidade de picos sistólicos (VPS) para as artérias

Tabela 4 - Média e desvio padrão (dp) obtidos dos diâmetros e das velocidades de pico sistólico (vps) e da velocidade diastólica final (vdf) da artéria aorta (aao). São Paulo, SP, Brasil. $n$ = número de amostra.

\begin{tabular}{lccc}
\hline $\mathrm{N}=25$ & AAO diâmetro $(\mathrm{cm})$ & AAO VPS $\left(\mathrm{cm} \mathrm{s}^{-1}\right)$ & AAO VDF $\left(\mathrm{cm} \mathrm{s}^{-1}\right)$ \\
\hline Média & 0,38 & 53,17 & 20,73 \\
DP & 0,04 & 13,46 & 7,17 \\
Mínimo & 0,28 & 37,29 & 8,74 \\
Mediana & 0,38 & 51,80 & 18,96 \\
Máximo & 0,46 & 83,77 & 39,14 \\
\hline
\end{tabular}

Ciência Rural, v.39, n.4, jul, 2009. 
renais foram de VPS 41,17 $\pm 9,40 \mathrm{~cm} \mathrm{~s}^{-1}$. Já os valores de velocidade diastólica final (VDF) foram de $19,14 \pm 5,50 \mathrm{~cm}$ $\mathrm{s}^{-1}$, e o IR foi de $0,53 \pm 0,07$. Para a aorta, foram obtidos os valores de VPS de $53,17 \pm 13,46 \mathrm{~cm} \mathrm{~s}^{-1}$ e de média de

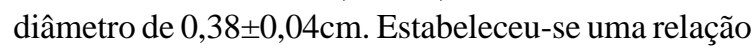
entre as velocidades de picos sistólicos da artéria renal e da aorta com o valor mínimo de 0,38 e o máximo de 1,52 .

\section{COMITÊ DE ÉTICA E BIOÉTICA}

Projeto aprovado sob o número 1391/2006 pela Comissão de Ética para a Análise de Projetos de Pesquisa do Instituto de Radiologia do Hospital das Clínicas da Faculdade de Medicina da Universidade de São Paulo.

\section{REFERÊNCIAS}

BARR,F.J. Evaluation of ultrasound as a method for assessing renal size in the dog. Journal of Small Animal Practice v.31, p.174-179, 1990.

BARR,F.J. et al. Ultrasonographic measurement of normal renal parameters. Journal of Small Animal Practice v.31, p.180-184, 1990.

BROWN, S et al. Guidelines for the identification, evaluation and management of systemic hypertension in dogs and cats. Journal of Veterinary Internal Medicine v. 21, p. 542558, 2007.

CERRI,G.G. et al. Avaliação do Doppler Renal. In: DOPPLER. São Paulo, SP: Sarvirer, 1998. Cap.7, p.131-154.

DYCE,K.M. et al. Tratado de anatomia veterinária. 2.ed. Rio de Janeiro: Guanabara Koogan, 1997. 663p.

JAIN,N.C. Comparative hematology of common domestic animals. In: ___. Essentials of veterinary hemotologin. Philadelphia: Lea \& Febiger, 1993. p.19-53

KANEKO, J.J. et al. Clinical biochemistry of domestic animals. 5.ed. San Diego, California: editor??. 1997. Appendix, p.895-903.
MELO, M.B. et al. Dopplerfluxometria das artérias renais: valores normais das velocidades sistólica e diastólica e do índice resistivo nas artérias renais principais. Arquivos Brasileiros de Medicina Veterinária e Zootecnia, v.58, n.4, p.691693, 2006. Disponível em: <http://www.scielo.br/ scielo.ph p ? script = s ci_art text \& pid = S 0102 $09352006000400040 \& \operatorname{lng}=\mathrm{en} \& \mathrm{nrm}=\mathrm{iso} \& \operatorname{lng}=\mathrm{pt}>$. Doi: 10.1590/S0102-09352006000400040.

NEWLL, S.M. et al. Scintigraphic, sonographic and histologic evaluation of renal autotransplantation in cats. American Journal of Veterinary Research, v.60, n.6, p.775-779, 1999.

NYLAND, T.G. et al. Diagnosis of urinary tract obstruction in dogs using duplex Doppler ultrasonography. Veterinary Radiology Ultrasound, v.34, n.5, p.384-352, 1993.

PLATT, J.F. Doppler Ultrasound of the kidney. Seminars in Ultrasound, CT and MRI, v.18, n.1, p. 22-32, 1997. Disponível em: <http://www.sciencedirect.com/ science?_ob=ArticleURL\&_udi=B 75M2-4D04XPJ$11 \&$ _user $=687358 \&$ \&_doc $=1 \&$ \& fmt $=$ \&_orig $=$ search \&_sort $=$ d \& vi e w = c \&_a c c t $=$ C $000037899 \&$ _version $=1 \&$ \& u r l V e r s i o n $=0 \&$ _ u s e r i d $=6873$ 58\&md5 $=5844 b 02 \mathrm{e} 1190 \mathrm{e} 91 \mathrm{~d} 19 \mathrm{c} 96219676 \mathrm{~b} 12 \mathrm{bf}>$. Doi: 10.1016/S0887-2171(97)90035-4.

POLLARD,R. et al. Ultrasonographic evaluation of renal autografts in normal cats. Veterinary Radiology and Ultrasound, v.40, n.4, p.380-385, 1999. Disponível em: <http://www3.interscience.wiley.com/journal/119097579/ abstract?CRETRY $=1 \&$ SRETRY $=0>$. Doi: $10.1111 / \mathrm{j} .1740$ 8261.1999.tb02130.x.

RIVERS,B.J. et al. Duplex Doppler estimation of resistive index in arcuate arteries of sedated, normal female dogs: implications for use in diagnosis of renal failure. Journal of American Animal Hospital Association, v.33, p.69-76, 1997.

RIVERS,B.J. et al. Duplex Doppler estimation of Pourcelot resistive index in arcuate arteries of sedated normal cats. Journal of Veterinary Internal Medicine, v.10, n.1, p.2833, 1996.

RIVERS, B.J. et al. Duplex Doppler estimation of intrarenal Pourcelot resistive index in dogs and cats with renal disease. Journal of Veterinary Internal Medicine, v.11, n.4, p.250260, 1997. 